The vacuum excitation and squeezing properties of two quantum oscillators with delta-kicked interactions

This article has been downloaded from IOPscience. Please scroll down to see the full text article.

2001 J. Phys. A: Math. Gen. 343429

(http://iopscience.iop.org/0305-4470/34/16/311)

View the table of contents for this issue, or go to the journal homepage for more

Download details:

IP Address: 129.123.125.21

The article was downloaded on $23 / 09 / 2011$ at $18: 27$

Please note that terms and conditions apply. 


\title{
The vacuum excitation and squeezing properties of two quantum oscillators with delta-kicked interactions
}

\author{
Jeong-Young Ji and David Peak \\ Department of Physics, Utah State University, Logan, UT 84322, USA \\ E-mail: jyji@cc.usu.edu and peakd@cc.usu.edu
}

Received 13 March 2000, in final form 11 January 2001

\begin{abstract}
The vacuum excitation and squeezing of two harmonic oscillators with deltakicked interactions for four types of elementary coupling are studied. The exact quantum motion for the Heisenberg operators and the explicit form of squeezing operators are found. The variances are calculated to study the squeezing properties for position, momentum and generalized quadrature operators.
\end{abstract}

PACS numbers: 0365,4250

\section{Introduction}

Two coupled harmonic oscillators with parametric excitations comprise an especially suitable system for studying the phenomena of zero-point excitation and squeezing of coherent states $[1,2]$. The correlated squeezed states of two permanently coupled oscillators with delta-kicked frequencies have been studied using a phase-space approach in [3]. Senitzky [4] has studied the effects on zero-point energy in elementary systems using a model consisting of two permanently coupled oscillators with four types of interaction (rotating-wave coupling, counter-rotating-wave coupling, dipole-dipole coupling and elastic-attraction coupling). There the interactions were assumed to be turned on at one time and remain constant thereafter. Senitzky found that all couplings except the rotating wave produce a lower zero-point energy than for the decoupled system, thus leading to a van der Waals attraction.

In this paper, we consider a different system of two oscillators with delta-kicked interactions of the four couplings mentioned above as exactly solvable models. These models are appropriate for describing a scattering process of two oscillators where the two oscillators are decoupled both before and after the interaction, for example, for approximately describing vibration modes of two colliding molecules. If the time interval of scattering process is relatively small compared to a timescale of interest, the delta-kicked interaction may be a good approximation. On the other hand, the study of squeezing properties of oscillator systems have long played important roles in studying particle creation (vacuum excitation) in quantum field theory [5] as well as in studying nonclassical effects of photon statistics in quantum optics [6]. The purpose of this work is to study the vacuum excitations and squeezing properties produced 
by the four couplings. In section 2, we introduce two harmonic oscillators with general couplings and find the exact quantum solution. In section 3 , we study the vacuum excitation and the squeezing for each coupling. The last section is devoted to a discussion.

\section{Coupled quantum oscillators with delta-kicked interaction}

Consider a system of two oscillators interacting via delta-kicked interactions. The Hamiltonian of this system is described by

$$
H=H_{1}+H_{2}+H_{\mathrm{I}}
$$

where

$$
H_{j}=\frac{1}{2 M_{j}} P_{j}^{2}+\frac{M_{j} \omega_{j}^{2}}{2} Q_{j}^{2}
$$

for $j=1,2$. We first introduce dimensionless position and momentum operators

$$
\begin{aligned}
& q_{j}=\sqrt{\frac{M_{j} \omega_{j}}{\hbar}} Q_{j} \\
& p_{j}=\frac{1}{\sqrt{M_{j} \hbar \omega_{j}}} P_{j}
\end{aligned}
$$

that satisfy the commutation relation $[q, p]=\mathrm{i}$. In terms of these new variables, the free oscillator Hamiltonians become

$$
H_{j}=\frac{1}{2} \hbar \omega_{j}\left(p_{j}^{2}+q_{j}^{2}\right)=\frac{1}{2} \hbar \omega_{j}\left(a_{j}^{\dagger} a_{j}+\frac{1}{2}\right)
$$

where the annihilation and creation operators are

$$
a_{j}=\left(q_{j}+\mathrm{i} p_{j}\right) / \sqrt{2} \quad a_{j}^{\dagger}=\left(q_{j}-\mathrm{i} p_{j}\right) / \sqrt{2} .
$$

In other words,

$$
\left(\begin{array}{l}
q_{1}(t) \\
p_{1}(t) \\
q_{2}(t) \\
p_{2}(t)
\end{array}\right)=D\left(\begin{array}{l}
a_{1}(t) \\
a_{1}^{\dagger}(t) \\
a_{2}(t) \\
a_{2}^{\dagger}(t)
\end{array}\right)
$$

where

$$
D=\left(\begin{array}{cccc}
\frac{1}{\sqrt{2}} & \frac{1}{\sqrt{2}} & 0 & 0 \\
\frac{-\mathrm{i}}{\sqrt{2}} & \frac{\mathrm{i}}{\sqrt{2}} & 0 & 0 \\
0 & 0 & \frac{1}{\sqrt{2}} & \frac{1}{\sqrt{2}} \\
0 & 0 & \frac{-\mathrm{i}}{\sqrt{2}} & \frac{\mathrm{i}}{\sqrt{2}}
\end{array}\right) .
$$

For the interaction Hamiltonian, we let

$$
H_{\mathrm{I}}=\hbar \delta(t)\left[\lambda_{1} q_{1}^{2}+\lambda_{2} q_{2}^{2}+2 \lambda_{q} q_{1} q_{2}+2 \lambda_{p} p_{1} p_{2}\right] .
$$

The Heisenberg equations of motion for the quantum position and momentum operators are as follows:

$$
\begin{aligned}
& \dot{q}_{1}=\omega_{1} p_{1}+2 \delta(t) \lambda_{p} p_{2} \\
& \dot{p}_{1}=-\omega_{1} q_{1}-2 \delta(t)\left(\lambda_{1} q_{1}+\lambda_{q} q_{2}\right) \\
& \dot{q}_{2}=\omega_{2} p_{2}+2 \delta(t) \lambda_{p} p_{1} \\
& \dot{p}_{2}=-\omega_{2} q_{2}-2 \delta(t)\left(\lambda_{2} q_{2}+\lambda_{q} q_{1}\right) .
\end{aligned}
$$


Solutions of the autonomous equations of motion for $t<0$ and $t>0$ oscillate in time as

$$
\begin{aligned}
& q_{j}(t)=q_{j}\left(0^{ \pm}\right) \cos \omega_{j} t+\frac{p_{j}\left(0^{ \pm}\right)}{\omega_{j}} \sin \omega_{j} t \\
& p_{j}(t)=-\omega_{j} q_{j}\left(0^{ \pm}\right) \sin \omega_{j} t+p_{j}\left(0^{ \pm}\right) \cos \omega_{j} t
\end{aligned}
$$

for $j=1,2$. The $t<0$ amplitudes and the $t>0$ amplitudes are related by

$$
B\left(\begin{array}{l}
q_{1}\left(0^{+}\right) \\
p_{1}\left(0^{+}\right) \\
q_{2}\left(0^{+}\right) \\
p_{2}\left(0^{+}\right)
\end{array}\right)=C\left(\begin{array}{l}
q_{1}\left(0^{-}\right) \\
p_{1}\left(0^{-}\right) \\
q_{2}\left(0^{-}\right) \\
p_{2}\left(0^{-}\right)
\end{array}\right)
$$

where

$$
B=\left(\begin{array}{cccc}
1 & 0 & 0 & -\lambda_{p} \\
\lambda_{1} & 1 & \lambda_{q} & 0 \\
0 & -\lambda_{p} & 1 & 0 \\
\lambda_{q} & 0 & \lambda_{1} & 1
\end{array}\right)
$$

and

$$
C=\left(\begin{array}{cccc}
1 & 0 & 0 & \lambda_{p} \\
-\lambda_{1} & 1 & -\lambda_{q} & 0 \\
0 & \lambda_{p} & 1 & 0 \\
-\lambda_{q} & 0 & -\lambda_{1} & 1
\end{array}\right)
$$

The time evolutions of the associated annihilation and creation operators, from (6) and (11), are given in simple forms by

$$
a_{j}(t)= \begin{cases}a_{j}\left(0^{-}\right) \mathrm{e}^{-\mathrm{i} \omega_{j} t} & \text { for } t<0 \\ a_{j}\left(0^{+}\right) \mathrm{e}^{-\mathrm{i} \omega_{j} t} & \text { for } t>0\end{cases}
$$

where

$$
\left(\begin{array}{l}
a_{1}\left(0^{+}\right) \\
a_{1}^{\dagger}\left(0^{+}\right) \\
a_{2}\left(0^{+}\right) \\
a_{2}^{\dagger}\left(0^{+}\right)
\end{array}\right)=D^{-1} B^{-1} C D\left(\begin{array}{l}
a_{1}\left(0^{-}\right) \\
a_{1}^{\dagger}\left(0^{-}\right) \\
a_{2}\left(0^{-}\right) \\
a_{2}^{\dagger}\left(0^{-}\right)
\end{array}\right) .
$$

\section{Excitation of a ground state and squeezing properties of coherent states}

In this section we consider four types of coupling, four different conditions on the $\lambda$ in (8). Suppose that the oscillators are initially in the vacuum (ground) state, $|0\rangle$ (which is annihilated by both $a_{1}\left(0^{-}\right)$and $a_{2}\left(0^{-}\right)$). Excitation of the ground state resulting from the interaction can be obtained by evaluating the expectation value of the number operator for each oscillator after the interaction

$$
N_{j, \text { created }}=\left\langle 0\left|a_{j}^{\dagger}\left(0^{+}\right) a_{j}\left(0^{+}\right)\right| 0\right\rangle \quad(j=1,2) .
$$

Squeezing of states can be assessed by examining the value of the variance of the quadrature operators $X$

$$
\Delta X^{2}=\left\langle\alpha_{1} \alpha_{2}\left|X^{2}\right| \alpha_{1} \alpha_{2}\right\rangle-\left\langle\alpha_{1} \alpha_{2}|X| \alpha_{1} \alpha_{2}\right\rangle^{2}
$$

where $X$ can be $q_{1}, p_{1}, q_{2}, p_{2}$ or their linear combinations such as

$$
\begin{aligned}
X_{1} & =\frac{1}{\sqrt{2}}\left(q_{1}-p_{2}\right) & Y_{1} & =\frac{1}{\sqrt{2}}\left(p_{1}+q_{2}\right) \\
X_{2} & =\frac{1}{\sqrt{2}}\left(q_{2}-p_{1}\right) & Y_{2} & =\frac{1}{\sqrt{2}}\left(p_{2}+q_{1}\right) .
\end{aligned}
$$


These quadrature operators satisfy $\left[X_{j}, Y_{k}\right]=\mathrm{i} \delta_{j k}$ and $\left[X_{j}, X_{k}\right]=0=\left[Y_{j}, Y_{k}\right]$. In (17), the two-mode coherent state is

$$
\left|\alpha_{1}, \alpha_{2}\right\rangle=D_{1}\left(\alpha_{1}\right) D_{2}\left(\alpha_{2}\right)|0\rangle
$$

where $D_{j}\left(\alpha_{j}\right)=\mathrm{e}^{\alpha_{j} a_{j}^{\dagger}\left(0^{-}\right)-\alpha_{j}^{*} a_{j}\left(0^{-}\right)}(j=1,2)$.

\subsection{Rotating-wave coupling}

Let us first consider the interaction

$$
H_{\mathrm{I}}=2 \hbar g \delta(t)\left(q_{1} q_{2}+p_{1} p_{2}\right)
$$

which, for $\omega_{1} \approx \omega_{2}$, has only slowly varying terms. By setting $\lambda_{q}=\lambda_{p}=g$ and $\lambda_{1}=\lambda_{2}=0$ from (15), we have

$$
\begin{aligned}
& a_{1}\left(0^{+}\right)=\frac{1-g^{2}}{1+g^{2}} a_{1}\left(0^{-}\right)-\frac{2 \mathrm{i} g}{1+g^{2}} a_{2}\left(0^{-}\right) \\
& a_{2}\left(0^{+}\right)=-\frac{2 \mathrm{i} g}{1+g^{2}} a_{1}\left(0^{-}\right)+\frac{1-g^{2}}{1+g^{2}} a_{2}\left(0^{-}\right) .
\end{aligned}
$$

This time evolution via delta-kicked interaction can be described by the following unitary, squeezing-like operator (hereafter $j=1,2$ and $a_{j}$ denotes $a_{j}\left(0^{-}\right)$, unless otherwise stated)

$$
S_{\mathrm{r}}=\mathrm{e}^{r^{*} a_{1} a_{2}^{\dagger}-r a_{1}^{\dagger} a_{2}}
$$

as

$$
\begin{aligned}
& S_{\mathrm{r}}^{\dagger} a_{1} S_{\mathrm{r}}=\cos |r| a_{1}-\sin |r| \mathrm{e}^{\mathrm{i} \theta_{r}} a_{2} \\
& S_{\mathrm{r}}^{\dagger} a_{2} S_{\mathrm{r}}=\sin |r| \mathrm{e}^{-\mathrm{i} \theta_{r}} a_{1}+\cos |r| a_{2}
\end{aligned}
$$

where $r=|r| \mathrm{e}^{\mathrm{i} \theta_{r}}, g=\tan \frac{|r|}{2}$ and $\theta_{r}=\frac{\pi}{2}$.

For this interaction there is no vacuum excitation

$$
N_{j, \text { created }}=0 .
$$

The variances

$$
\Delta q_{j}^{2}=\Delta p_{j}^{2}=\frac{1}{2}
$$

satisfy the minimal uncertainty relation before and after interaction

$$
\Delta q_{j} \Delta p_{j}=\frac{1}{2}
$$

and, hence, there is no state squeezing in the two individual modes. The variances of generalized quadratures

$$
\Delta X_{j}^{2}=\Delta Y_{j}^{2}=\frac{1}{2}
$$

also show no squeezing.

\subsection{Counter-rotating-wave coupling}

Now let us consider the following interaction:

$$
H_{\mathrm{I}}=2 \hbar g \delta(t)\left(q_{1} q_{2}-p_{1} p_{2}\right)
$$

which has only fast-varying terms. By setting $\lambda_{q}=-\lambda_{p}=g$ and $\lambda_{1}=\lambda_{2}=0$ from (15), we have

$$
\begin{aligned}
& a_{1}\left(0^{+}\right)=\frac{1+g^{2}}{1-g^{2}} a_{1}\left(0^{-}\right)-\frac{2 \mathrm{i} g}{1-g^{2}} a_{2}^{\dagger}\left(0^{-}\right) \\
& a_{2}\left(0^{+}\right)=-\frac{2 \mathrm{i} g}{1-g^{2}} a_{1}^{\dagger}\left(0^{-}\right)+\frac{1+g^{2}}{1-g^{2}} a_{2}\left(0^{-}\right)
\end{aligned}
$$


We introduce the two-mode squeezing operator [7]

$$
S_{\mathrm{c}}(r)=\mathrm{e}^{r^{*} a_{1} a_{2}-r a_{1}^{\dagger} a_{2}^{\dagger}}
$$

which transforms $a_{1}$ and $a_{2}$ to

$$
\begin{aligned}
& S_{\mathrm{c}}^{\dagger} a_{1} S_{\mathrm{c}}=\cosh |r| a_{1}-\sinh |r| \mathrm{e}^{\mathrm{i} \theta_{r}} a_{2}^{\dagger} \\
& S_{\mathrm{c}}^{\dagger} a_{2} S_{\mathrm{c}}=-\sinh |r| \mathrm{e}^{\mathrm{i} \theta_{r}} a_{1}^{\dagger}+\cosh |r| a_{2} .
\end{aligned}
$$

For $|g|<1$, this operator describes the time evolution of (29) with $g=\tanh \frac{|r|}{2}$ and $\theta=\frac{\pi}{2}$. The variances of quadratures

$$
\begin{aligned}
\Delta q_{j}^{2}=\Delta p_{j}^{2} & =\frac{1}{2}\left(\cosh ^{2}|r|+\sinh ^{2}|r|\right) \\
& =\frac{1}{2} \frac{1+6 g^{2}+g^{4}}{\left(1-g^{2}\right)^{2}}
\end{aligned}
$$

and uncertainty relations

$$
\Delta q_{j} \Delta p_{j}=\frac{1}{2} \frac{1+6 g^{2}+g^{4}}{\left(1-g^{2}\right)^{2}}
$$

there is no squeezing in the two individual modes, as is well known [8]. Instead, the variances of generalized quadratures (18)

$$
\Delta X_{j}^{2}=\frac{1}{2} \mathrm{e}^{2|r|} \quad \Delta Y_{j}^{2}=\frac{1}{2} \mathrm{e}^{-2|r|}
$$

which clearly show ideal squeezing $\left(\Delta X_{j} \Delta Y_{j}=1 / 2\right)$. Therefore, the coherent state (19) before interaction becomes the squeezed state after interaction. Finally the vacuum excitation is

$$
N_{j, \text { created }}=\sinh ^{2}|r|=\frac{4 g^{2}}{\left(1-g^{2}\right)^{2}}
$$

\subsection{Dipole-dipole coupling}

Our oscillator system for the interaction

$$
H_{\mathrm{I}}=4 \hbar g \delta(t) q_{1} q_{2}
$$

is described by setting $\lambda_{q}=2 g$ and $\lambda_{1}=\lambda_{2}=\lambda_{p}=0$ from (15). The time evolutions are

$$
\begin{aligned}
& a_{1}\left(0^{+}\right)=a_{1}\left(0^{-}\right)-2 \mathrm{i} g\left[a_{2}\left(0^{-}\right)+a_{2}^{\dagger}\left(0^{-}\right)\right] \\
& a_{2}\left(0^{+}\right)=a_{2}\left(0^{-}\right)-2 \mathrm{i} g\left[a_{1}\left(0^{-}\right)+a_{1}^{\dagger}\left(0^{-}\right)\right] .
\end{aligned}
$$

As in the previous interactions, the unitary operator

$$
S_{\mathrm{d}}=\mathrm{e}^{-\mathrm{i} 4 g q_{1} q_{2}}=\mathrm{e}^{-\mathrm{i} 2 g\left(a_{1} a_{2}+a_{1}^{\dagger} a_{2}^{\dagger}+a_{1} a_{2}^{\dagger}+a_{1}^{\dagger} a_{2}\right)}
$$

gives the time evolution (37). The variances are

$$
\Delta q_{j}^{2}=\frac{1}{2} \quad \Delta p_{j}^{2}=\frac{1}{2}+8 g^{2}
$$

and the uncertainty relations

$$
\Delta q_{j} \Delta p_{j}=\frac{1}{2} \sqrt{1+16 g^{2}} .
$$

From the variances

$$
\begin{aligned}
& \Delta X_{j}^{2}=\frac{1}{2}+2 g+4 g^{2} \\
& \Delta Y_{j}^{2}=\frac{1}{2}-2 g+4 g^{2}
\end{aligned}
$$

we see the squeezing in the quadrature $Y_{1}$ and $Y_{2}$ when $0<g<\frac{1}{2}$. The vacuum excitation is given by

$$
N_{j, \text { created }}=4 g^{2} \text {. }
$$




\subsection{Elastic-attraction coupling}

For the interaction

$$
H_{\mathrm{I}}=\hbar g \delta(t)\left(q_{1}-q_{2}\right)^{2}
$$

by setting $\lambda_{1}=\lambda_{2}=-\lambda_{q}=g$ and $\lambda_{p}=0$ from (15), we have

$$
\begin{aligned}
& a_{1}\left(0^{+}\right)=(1-\mathrm{i} g) a_{1}-\mathrm{i} g a_{1}^{\dagger}+\mathrm{i} g a_{2}+\mathrm{i} g a_{2}^{\dagger} \\
& a_{2}\left(0^{+}\right)=(1-\mathrm{i} g) a_{2}-\mathrm{i} g a_{2}^{\dagger}+\mathrm{i} g a_{1}+\mathrm{i} g a_{1}^{\dagger}
\end{aligned}
$$

which are evolved in time by

$$
S_{\mathrm{e}}=\mathrm{e}^{-\mathrm{i} g\left(q_{1}-q_{2}\right)^{2}} .
$$

The variances are

$$
\Delta q_{j}^{2}=\frac{1}{2} \quad \Delta p_{j}^{2}=\frac{1}{2}+4 g^{2}
$$

and the uncertainties are

$$
\Delta q_{j} \Delta p_{j}=\frac{1}{2} \sqrt{1+8 g^{2}} .
$$

Quite different from the dipole-dipole coupling, there are squeezings in $X_{1}$ and $X_{2}$ when $0<g<\frac{1}{2}$

$$
\Delta X_{j}^{2}=\frac{1}{2}-g+2 g^{2} \quad \Delta Y_{j}^{2}=\frac{1}{2}+g+2 g^{2} .
$$

Finally, the vacuum excitation is as follows:

$$
N_{j, \text { created }}=2 g^{2} \text {. }
$$

\section{Discussion}

We considered two coupled harmonic oscillators with four types of delta-kicked interaction: rotating-wave coupling, counter-rotating-wave coupling, dipole-dipole coupling and elasticattraction coupling. Since we have found the time evolutions of annihilation and creation operators, it is trivial to calculate coordinate dispersions, momentum dispersions, correlations, squeezing parameters and correlation coefficients. We have calculated the vacuum excitation (excitation of ground state or particle creation) and squeezing of the quantum coherent states. In this paper we have only considered an ideal case and ignored dissipation. In order to take a real situation such as molecule collisions, we have to consider a series of delta-kicks with irregular time intervals (for regular time intervals in a single oscillator, see [9,10]) and either use the quantum Fokker-Planck equation [11] or couple the system to a dissipative heat bath [12]. This generalization will be considered in our future work.

All couplings but the rotating-wave coupling give vacuum excitation and squeezing. This result is very interesting in relation to the conclusion of [4], where all couplings but the rotating wave lead to the presence of a van der Waals attraction. In fact, the rotating-wave coupling and the counter-rotating-wave coupling are closely related to nonlinear interactions in quantum optics. It is well known that the vacuum is not excited by a rotating-wave coupling. As physical examples of the other two couplings, the elastic coupling is a standard textbook example of an oscillator chain and the dipole-dipole coupling describes the interaction of two polar molecules. Our model calculation may be applied to the excitation of molecule vibration when they interact for a very short time and the two-mode squeezing in the quantum optics. The well known squeezing operator $S_{\mathrm{c}}$ keeps the minimal uncertainty relations $\Delta X_{j} \Delta Y_{j}=\frac{1}{2}$ while squeezing the $\Delta Y_{j}$. The new squeezing operators $S_{\mathrm{d}}$ and $S_{\mathrm{e}}$ squeeze the quadrature $Y_{j}$ and $X_{j}$, respectively. Since a small oscillation from equilibrium in any physical system is modelled by a harmonic oscillator, our interaction models may be applied to many physical situations when the interactions between oscillators are considered. 


\section{References}

[1] Kim Y S and Man'ko V I 1991 Phys. Lett. A 157226

[2] Lo C F 1992 Phys. Lett. A 162299

[3] Man'ko O V and Yeh L 1994 Phys. Lett. A 189268

[4] Senitzky I R 1998 Phys. Rev. A 5740

[5] Hu B L, Kang G and Matacz A 1994 Int. J. Mod. Phys. A 9991

[6] Kolobov M I 1999 Rev. Mod. Phys. 711539

[7] Yuen H P 1976 Phys. Rev. A 132226

[8] Barnett S M and Knight P L 1985 J. Opt. Soc. Am. B 2467

[9] Dodonov V V 1996 Phys. Lett. A 21427

[10] Gardiner S A, Cirac J I and Zoller P 1997 Phys. Rev. Lett. 794790

[11] Dodonov V V and Man'ko O V 1985 Physica A 130353

[12] Ford G W, Lewis J T and O'Connell R F 1988 Phys. Rev. A 374419 